\title{
P01-8-46 \\ Poster session
}

\section{The role of nutraceuticals in the chemoprevention of the colorectal neoplasm}

\author{
Mehdi Bouhlel, Mehdi Dridi, Abderrahman Bouraoui
}

Department of Pharmacology, College of Pharmacy, University of Monastir TUNISIA, Tunisia

Background:

The colorectal neoplasm is considered as the third most common cause of cancer-related death in the world and its incidence has continually increased mainly in industrialized countries. Identifying effective cancer prevention strategies to inhibit the growth and progression of the colorectal neoplasm is an essential step to reduce the incidence and the mortality of this malignancy.

Methods:

Our work consists on literature review during the last five years in order to evaluate the role of nutraceuticals in colorectal cancer prevention.

We proceeded with a synthesis of the data results of experimental studies in vitro and clinical studies in vivo with studying the molecular mechanism of action in the chemoprevention anti colorectal neoplasm of :probiotics, curcumin,vitamin D, calcium, carotenoids and folate.

Results

The results of our study demonstrate the role of those substances in the chemoprevention anti colorectal neoplasm with a comprehension of the mechanism of action especially with the curcumin that demonstrates the best efficacy and safety however vitamin D and folate are still contreversial. We must be aware about the safety and the efficacy of such substances under some circumstances.

Conclusion:

More studies in advanced level are required in order to deeply understand the efficacy of the nutraceuticals as a chemopreventive agent against the colorectal neoplasm with the determination of the doses required to obtain the chemopreventive role and why not setting a chemopreventive protocols using these substances. 